\title{
1 Theory of the Quantum Hall Effect in Quasi-One-Dimensional Conductors
}

\author{
Victor M. Yakovenko \\ Department of Physics, University of Maryland, College Park, MD 20742-4111, \\ USA, http://www2.physics.umd.edu/〜 yakovenk
}

This Chapter reviews the theory of the quantum Hall effect (QHE) in quasione-dimensional (Q1D) conductors. It is primarily based on the author's papers $\begin{array}{llll}1 & 2 & 3 & 4\end{array}$. The QHE in Q1D conductors is closely related to the magneticfield-induced spin-density wave (FISDW) observed in these materials. The theory of the FISDW is reviewed in this book by L. Gor'kov, by M. Héritier, by A. Lebed, and by S. Haddad et al. The FISDW experiment is reviewed by P. Chaikin et al. and by V. Pudalov and A. Kornilov.

\subsection{Introduction to quasi-one-dimensional conductors}

Organic metals of the (TMTSF) $)_{2} \mathrm{X}$ family, where TMTSF is tetramethyltetraselenafulvalene, and $\mathrm{X}$ is an inorganic anion such as $\mathrm{PF}_{6}$, are Q1D crystals consisting of parallel conducting chains formed by the organic molecules TMTSF. The chains direction is denoted as $\boldsymbol{a}$ or $x$. The interchain coupling is much weaker in the $\boldsymbol{c}(z)$ direction than in the $\boldsymbol{b}(y)$ direction, so the chains form weakly coupled two-dimensional (2D) layers. In a simple model, the electron dispersion $\varepsilon(\boldsymbol{k})$ in these materials is described by a tight-binding model representing tunneling between the TMTSF molecules

$$
\varepsilon=2 t_{a} \cos \left(k_{x} a\right)+2 t_{b} \cos \left(k_{y} b\right)+2 t_{a} \cos \left(k_{z} d\right)+\ldots,
$$

where $\boldsymbol{k}=\left(k_{x}, k_{y}, k_{c}\right)$ is the electron wave vector. The intermolecule distances are $a=0.73 \mathrm{~nm}, b=0.77 \mathrm{~nm}$, and $d=1.35 \mathrm{~nm} \mathrm{[5]}$, and we approximate the triclinic crystal structure by the orthogonal one. The electron tunneling amplitudes $t_{a} \gg t_{b} \gg t_{c}$ are estimated as $250 \mathrm{meV}, 25 \mathrm{meV}$, and $1.5 \mathrm{meV}$ [5]. The band (1.1) is quarter-filled ${ }^{1}$ by holes, because each anion $\mathrm{X}^{-}$takes one electron. The Fermi surface is open and consists of two disconnected sheets with $k_{x}$ close to $\pm k_{\mathrm{F}}$, where $k_{\mathrm{F}}=\pi / 4 a$ is the Fermi momentum along the chains. In the vicinity of the Fermi surface, we can linearize the longitudinal electron dispersion $\varepsilon_{\|}\left(k_{x}\right)$. Measuring $\varepsilon$ from the Fermi energy and neglecting $t_{c}$, we can approximate (1.1) as

\footnotetext{
${ }^{1}$ We ignore weak dimerization of the TMTSF molecules [5], which is not essential for our consideration.
} 


$$
\varepsilon_{ \pm}=\varepsilon_{\|}\left(k_{x}\right)+\varepsilon_{\perp}\left(k_{y} b\right)= \pm \hbar v_{\mathrm{F}}\left(k_{x} \mp k_{\mathrm{F}}\right)+2 t_{b} \cos \left(k_{y} b\right)+2 t_{b}^{\prime} \cos \left(2 k_{y} b\right),
$$

where the signs \pm correspond to the two sheets of the Fermi surface, $v_{\mathrm{F}}=$ $\partial \varepsilon_{\|} / \hbar \partial k_{x} \approx 10^{5} \mathrm{~m} / \mathrm{s}[6]$ is the Fermi velocity, and $\hbar$ is the Planck constant. In this Chapter, we study the in-plane Hall conductivity $\sigma_{x y}$ per one layer in a magnetic field $\boldsymbol{B}$ applied perpendicular to the $\boldsymbol{a}-\boldsymbol{b}$ layers. The interlayer coupling $t_{c}$ is not essential for this consideration, so most of the theory is presented for just one 2D layer, as in (1.2). The tunneling amplitude $t_{b}^{\prime}$ to the next-nearest chain in (1.2) is important for the FISDW theory.

\subsection{Hall effect in the normal state}

Let us briefly discuss the Hall effect in the normal state of (TMTSF) $)_{2} \mathrm{X}$. The textbook formula says that the Hall coefficient is $R_{\mathrm{H}}=1 / n e c$, where $n$ is the carrier concentration per one layer, $e$ is the electron charge, and $c$ is the speed of light. However, the linearized model (1.2) gives $R_{\mathrm{H}}=0$, because of the electron-hole symmetry. A non-zero result is obtained by taking into account the curvature $\beta$ of the longitudinal electron dispersion $[7,8,9]$ :

$$
R_{\mathrm{H}}^{(\mathrm{n})}=\frac{\beta}{n e c}, \quad \beta=\frac{k_{\mathrm{F}}}{v_{\mathrm{F}}} \frac{\partial^{2} \varepsilon_{\|}}{\partial k_{x}^{2}} .
$$

For the quarter-filled tight-binding band (1.1) in (TMTSF) $)_{2} \mathrm{X}, n=1 / 2 a b$ and $\beta=\pi / 4$ [7, 8, 9], as opposed to $\beta=1$ for the conventional parabolic dispersion. The experimentally measured $R_{\mathrm{H}}^{(\mathrm{n})}$ [10,11] is in overall agreement with (1.3), but it also exhibits some puzzling temperature dependence 912].

The Hall resistivity $\rho_{x y}=R_{\mathrm{H}}^{(\mathrm{n})} B=\beta\left(h / e^{2}\right)\left(2 a b B / \phi_{0}\right)$ is small and not quantized. This is because, for any realistic $B$, the magnetic flux through the area $2 a b$ per one carrier is much smaller that the flux quantum $\phi_{0}=h c / e$, so the Landau filling factor is very high, of the order of $10^{2}-10^{3}$.

\subsection{Introduction to the quantum Hall effect in the FISDW state}

When a strong magnetic field $B$ is applied in the $z$ direction perpendicular to the layers, the (TMTSF) $)_{2} \mathrm{X}$ materials experience a cascade of phase transitions between the so-called magnetic-field-induced spin-density-wave states. These are true thermodynamic phase transitions, observed in specific heat [13, 14, magnetization [15], NMR [16, and virtually all other physical quantities [17 18. A detailed theory of the FISDW is presented in other chapters of this book, as well as in the book [5] and in the review volume [19]. According to the theory [20 21 22 23], the electron spin density in the FISDW state develops spontaneous modulation with the wave vector 


$$
\boldsymbol{Q}=\left(Q_{x}, Q_{y}, Q_{z}\right), \quad Q_{x}=2 k_{\mathrm{F}}-N G, \quad G=e b B / \hbar c, \quad E_{B}=\hbar v_{\mathrm{F}} G,
$$

where $G$ is the characteristic wave vector of the magnetic field, and $N$ is an integer number (positive or negative). The magnetic length $l_{x}=2 \pi / G$ is defined so that the magnetic flux through the area bounded by $l_{x}$ and by the interchain distance $b$ is equal to the flux quantum: $B l_{x} b=\phi_{0}$. Notice that $G \ll k_{\mathrm{F}}$, because $B a b \ll \phi_{0}$ for realistic magnetic fields. We also introduced the characteristic energy $E_{B}$ of the magnetic field in (1.4).

The difference between $Q_{x}$ and $2 k_{\mathrm{F}}$ in (1.4) plays crucial role for the Hall effect. Let us calculate $\sigma_{x y}$ in the FISDW state by naively counting the number of available carriers 20. The FISDW with the wave vector $\boldsymbol{Q}$ (1.4) hybridizes the $\pm k_{\mathrm{F}}$ sheets of the Fermi surface and opens an energy gap. Some electron and hole pockets form above and below the energy gap because of imperfect nesting. The effective carrier concentration $n$ is determined by their total area in the momentum space. This is the area between the two sheets of the Fermi surface (1.2) $k_{x}^{ \pm}\left(k_{y}\right)= \pm k_{\mathrm{F}} \mp \varepsilon_{\perp}\left(k_{y}\right) / v_{\mathrm{F}}$, one of them shifted by the wave vector $\left(Q_{x}, Q_{y}\right)$ :

$$
\begin{aligned}
n & =\frac{2}{2 \pi} \int_{0}^{2 \pi / b} \frac{\mathrm{d} k_{y}}{2 \pi}\left\{k_{x}^{+}\left(k_{y}\right)-\left[k_{x}^{-}\left(k_{y}-Q_{y}\right)+Q_{x}\right]\right\} \\
& =\frac{2}{2 \pi} \int_{0}^{2 \pi / b} \frac{\mathrm{d} k_{y}}{2 \pi}\left(2 k_{\mathrm{F}}-Q_{x}+\frac{\varepsilon_{\perp}\left(k_{y}-Q_{y}\right)-\varepsilon_{\perp}\left(k_{y}\right)}{v_{\mathrm{F}}}\right) .
\end{aligned}
$$

Here the factor 2 accounts for two spin projections. The integral of the last term in (1.5) vanishes for any $Q_{y}$, because

$$
\int_{0}^{2 \pi / b} \varepsilon_{\perp}\left(k_{y}\right) \mathrm{d} k_{y}=0,
$$

whereas (1.4) and the first term in 1.5) give $n=2 \mathrm{NeB} / \mathrm{hc}$. Substituting this expression in the textbook formula $\sigma_{x y}=n e c / B$, we find

$$
\sigma_{x y}=2 N e^{2} / h .
$$

Formula (1.7) was also derived in 24 using the Středa formula.

Equation (1.7) represents the integer quantum Hall effect (QHE). The Hall effect becomes quantized, because the FISDW (1.4) eliminates most of the carriers, but leaves $N$ fully occupied Landau levels in the remaining pockets [25]. With the increase of $B$, the system experiences a cascade of phase transitions between the FISDW states with different numbers $N$. This produces a series of the quantum Hall plateaus (1.7) separated by phase transitions, as indeed observed experimentally in (TMTSF) $)_{2} \mathrm{X}[25,26,27,28$, 29, 30, 31 32 33 34. In a simple case, $N$ takes consecutive numbers $0,1,2$, ... with decreasing magnetic field $B$ [25, 26, 27, 28], but $N(B)$ may also show more complicated sequences, including sign changes [27, 29, 30, 31,32, 33 34. The bulk Hall conductivity is measured in experiment, and $\sigma_{x y}$ per one layer 
is obtained by dividing by the number of conducting layers. The latter may depend on current distribution, so the absolute values of $\sigma_{x y}$ are not obtained very precisely [31, but the relative values of $\sigma_{x y}$ do correspond to integer numbers. In the FISDW state, $\sigma_{x y} \gg \sigma_{x x}, \sigma_{y y}$, as expected for the QHE 27, $29,30,33,34$.

The derivation of (1.7) presented above is simple, but not quite satisfactory. In the FISDW state, there are multiple gaps in the energy spectrum of electrons, including a gap at the Fermi level, so the notion of metallic electron and hole pockets is not quite meaningful. Moreover, the magnetic energy $E_{B}$ is typically greater than the FISDW gap $\Delta$, so there is strong magnetic breakdown between the pockets, and the semiclassical Landau quantization using closed electron orbits is not well defined. After briefly summarizing the FISDW theory in Sect.1.4 we present a more rigorous derivation of the QHE as a topological invariant [1] in Sect.1.5 This formalism is also utilized for various generalizations presented in the subsequent Sections.

\subsection{Mathematical theory of the FISDW}

A magnetic field $B$ applied perpendicular to the layers can be introduced in the electron dispersion (1.2) via the Peierls-Onsager substitution $k_{y} \rightarrow$ $k_{y}-(e / \hbar c) A_{y}$ in the gauge $A_{y}=B x$. As a result, the transverse dispersion $\varepsilon_{\perp}\left(k_{y} b-G x\right)$ produces a periodic potential in the $x$ direction with the wave vector $G$ given by (1.4) 35 36]. Electrons also experience the periodic potential $\Delta_{0} \cos \left(Q_{x} x+Q_{y} y\right)$ from the FISDW. To find the energy spectrum, let us decompose the electron wave function $\psi\left(x, k_{y}\right)$ into the components $\psi_{+}\left(x, k_{y}\right)$ and $\psi_{-}\left(x, k_{y}\right)$ with the longitudinal momenta close to $\pm k_{\mathrm{F}}$ :

$$
\psi\left(x, k_{y}\right)=\psi_{+}\left(x, k_{y}\right) \mathrm{e}^{+\mathrm{i} k_{\mathrm{F}} x}+\psi_{-}\left(x, k_{y}\right) \mathrm{e}^{-\mathrm{i} k_{\mathrm{F}} x} .
$$

Introducing the two-component spinor $\left[\psi_{+}\left(x, k_{y}\right), \psi_{-}\left(x, k_{y}+Q_{y}\right)\right]$, we can write the electron Hamiltonian as a $2 \times 2$ matrix operating on this spinor

$$
\hat{H}=\left(\begin{array}{cc}
-\mathrm{i} v_{\mathrm{F}} \partial_{x}+\varepsilon_{\perp}\left(k_{y} b-G x\right) & \Delta_{0} \mathrm{e}^{\mathrm{i}\left(Q_{x}-2 k_{\mathrm{F}}\right) x} \\
\Delta_{0} \mathrm{e}^{-\mathrm{i}\left(Q_{x}-2 k_{\mathrm{F}}\right) x} & \mathrm{i} v_{\mathrm{F}} \partial_{x}+\varepsilon_{\perp}\left(k_{y} b+b Q_{y}-G x\right)
\end{array}\right) .
$$

Here the diagonal terms represent the electron dispersion (1.2) in the presence of a magnetic field. The off-diagonal terms represent the periodic potential of the FISDW (1.4). The wave vector $2 k_{\mathrm{F}}$ is subtracted from $Q_{x}$ because of the $\mathrm{e}^{ \pm \mathrm{i} k_{\mathrm{F}} x}$ factors introduced in (1.8). We do not write the spin indices explicitly in (1.8) and (1.9), because they are not essential for our consideration. Equations (1.8) and (1.9) can be applied to pairing between $\psi_{+}$and $\psi_{-}$with parallel or antiparallel spins, as discussed in more detail in [1.

Now let us make a phase transformation of the spinor components ${ }^{2}$

\footnotetext{
${ }^{2}$ This kind of transformation was first introduced in [35], which started development of the FISDW theory.
} 


$$
\left(\begin{array}{l}
\psi_{+} \\
\psi_{-}
\end{array}\right)=\left(\begin{array}{l}
\psi_{+}^{\prime} \exp \left[\left(\mathrm{i} / E_{B}\right) \int^{k_{y} b-G x} \varepsilon_{\perp}(\xi) \mathrm{d} \xi\right] \\
\psi_{-}^{\prime} \exp \left[-\left(\mathrm{i} / E_{B}\right) \int^{k_{y} b-G x} \varepsilon_{\perp}\left(Q_{y} b+\xi\right) \mathrm{d} \xi\right]
\end{array}\right),
$$

where $E_{B}$ is the characteristic magnetic energy given in (1.4). Substituting (1.10) into (1.9), we obtain a new Hamiltonian acting on the spinor $\left(\psi_{+}^{\prime}, \psi_{-}^{\prime}\right)$

$$
\hat{H}^{\prime}=\left(\begin{array}{ll}
-\mathrm{i} v_{\mathrm{F}} \partial_{x} & \tilde{\Delta}(x) \\
\tilde{\Delta}^{*}(x) & \mathrm{i} v_{\mathrm{F}} \partial_{x}
\end{array}\right) .
$$

As a result of the transformation (1.10), the $\varepsilon_{\perp}$ terms are removed from the diagonal in (1.11), but they re-appear in the off-diagonal terms

$$
\tilde{\Delta}(x)=\Delta_{0} \exp \left\{-\mathrm{i} N G x-\frac{\mathrm{i}}{E_{B}} \int^{k_{y} b-G x}\left[\varepsilon_{\perp}(\xi)+\varepsilon_{\perp}\left(Q_{y} b+\xi\right)\right] \mathrm{d} \xi\right\} .
$$

Since $\varepsilon_{\perp}\left(k_{y}\right)$ satisfies (1.6), the integral in (1.12) is a periodic function of $k_{y} b-G x$, so $\tilde{\Delta}(x)$ can be expanded in a Fourier series with coefficients $c_{m}$

$$
\tilde{\Delta}(x)=\Delta_{0} \mathrm{e}^{-\mathrm{i} N G x} \sum_{m} c_{m} \mathrm{e}^{\mathrm{i} m\left(k_{y} b-G x\right)} .
$$

Because the FISDW forms at a low transition temperature in high magnetic fields, it is appropriate to consider the limit $\Delta_{0} \ll E_{B}$, where the FISDW potential is much weaker than the magnetic energy. In this case, when (1.13) is substituted into (1.11), each periodic potential in the sum 1.13) can be treated separately and opens a gap $2\left|\Delta_{0} c_{m}\right|$ in the energy spectrum at the wave vectors $k_{x}= \pm\left[k_{\mathrm{F}}-(N+m) G / 2\right]$ [37,38. The electron mini-bands separated by the energy gaps can be interpreted as the broadened Landau levels, with the number of states in each mini-band proportional to $G \propto B$.

The term with $m=-N$ in (1.13) opens a gap at the Fermi level. This is possible only if $2 k_{\mathrm{F}}-Q_{x}$ is an integer multiple of $G$, i.e. there are $N$ minibands between the gaps at $k_{x}= \pm Q_{x} / 2$ and $k_{x}= \pm k_{\mathrm{F}}$. Let us focus on the gap at the Fermi level and omit the terms with $m \neq-N$ in the sum (1.13). In this single-gap approximation [37,38], the Hamiltonian (1.11) becomes the same as for a $1 \mathrm{D}$ density wave [39] with the effective amplitude $\Delta \mathrm{e}^{-\mathrm{i} \varphi}$ :

$$
\hat{H}^{\prime}=\left(\begin{array}{ll}
-\mathrm{i} v_{\mathrm{F}} \partial_{x} & \Delta \mathrm{e}^{-\mathrm{i} \varphi\left(k_{y}\right)} \\
\Delta^{*} \mathrm{e}^{\mathrm{i} \varphi\left(k_{y}\right)} & \mathrm{i} v_{\mathrm{F}} \partial_{x}
\end{array}\right), \quad \Delta=\Delta_{0} c_{-N}, \quad \varphi=N b k_{y} .
$$

The Hamiltonian (1.14) has the gapped energy spectrum

$$
E\left(p_{x}\right)=\sqrt{\left(v_{\mathrm{F}} p_{x}\right)^{2}+|\Delta|^{2}}, \quad p_{x}=\hbar\left(k_{x}-k_{\mathrm{F}}\right) .
$$

Notice that the off-diagonal terms in (1.14) have the phase $\varphi\left(k_{y}\right)$, which does not matter for the energy spectrum (1.15), but plays crucial role in the QHE. ${ }^{3}$

\footnotetext{
${ }^{3}$ The electron conductivity tensor for the FISDW state was calculated in [40], but quantized contribution to $\sigma_{x y}$ was lost.
} 
The Fourier coefficients $c_{m}$ (1.13) depend on $Q_{y}$ and the ratio of the tunneling amplitudes $t_{b}$ and $t_{b}^{\prime}$ (1.2) to the magnetic energy $E_{B}$. The values of $N$ and $Q_{y}$ in a FISDW state are selected in such a way as to maximize the coefficient $\left|c_{-N}\left(Q_{y}\right)\right|$ and, thus, the energy gap $\Delta$ (1.14) at the Fermi level for a given magnetic magnetic field. ${ }^{4}$ As magnetic field changes, the optimal values of $N$ and $Q_{y}$ change. Since the parameter $N$ must be integer in order to produce a gap at the Fermi level, it changes by discontinuous jumps, which produces a cascade of the FISDW transitions.

\subsection{Quantum Hall effect as a topological invariant}

Suppose an electric field $E_{y}$ is applied perpendicular to the chains. We can introduce it in the Hamiltonian (1.9) by the Peierls-Onsager substitution $k_{y} \rightarrow k_{y}-(e / \hbar c) A_{y}$ using the gauge $A_{y}=-E_{y} c t$, where $t$ is time. Then, the periodic potential $\varepsilon_{\perp}\left[k_{y} b-G(x-v t)\right]$ starts to move with the velocity $v=c E_{y} / B$. This motion induces some current $j_{x}$ along the chains, which constitutes the Hall effect. However, the FISDW periodic potential in the offdiagonal terms in (1.9) does not move, if the FISDW is pinned. The moving and non-moving periodic potentials are combined in the effective Hamiltonian (1.14), where the phase becomes time-dependent $\varphi=N b\left(k_{y}+e E_{y} t / \hbar\right)$. The time-dependent phase means that the effective 1D density wave (1.14) slides along the chains, carrying the Fröhlich current 39.

$$
j_{x}=\frac{2 e}{2 \pi b} \frac{\partial \varphi}{\partial t}=\frac{2 N e^{2}}{h} E_{y}=\sigma_{x y} E_{y} .
$$

Equation (1.16) represents the quantum Hall effect and agrees with (1.7). ${ }^{5}$

The Hall conductivity at zero temperature can be also expressed in terms of a topological invariant called the Chern number [45] 46]:

$$
\begin{gathered}
\sigma_{x y}=-\mathrm{i} \frac{e^{2}}{\hbar} \sum_{a} \int \frac{\mathrm{d} k_{x}}{2 \pi} \int \frac{\mathrm{d} k_{y}}{2 \pi}\left(\frac{\partial\left\langle\psi_{a}\right|}{\partial k_{x}} \frac{\partial\left|\psi_{a}\right\rangle}{\partial k_{y}}-\frac{\partial\left\langle\psi_{a}\right|}{\partial k_{y}} \frac{\partial\left|\psi_{a}\right\rangle}{\partial k_{x}}\right) \\
=-\mathrm{i} \frac{e^{2}}{\hbar} \sum_{a} \int \frac{\mathrm{d} k_{x}}{2 \pi} \int \frac{\mathrm{d} k_{y}}{2 \pi}\left[\frac{\partial}{\partial k_{x}}\left(\left\langle\psi_{a}\right| \frac{\partial\left|\psi_{a}\right\rangle}{\partial k_{y}}\right)-\frac{\partial}{\partial k_{y}}\left(\left\langle\psi_{a}\right| \frac{\partial\left|\psi_{a}\right\rangle}{\partial k_{x}}\right)\right] .
\end{gathered}
$$

Here $\left|\psi_{a}\left(k_{x}, k_{y}\right)\right\rangle$ are the normalized eigenvectors of the Hamiltonian. The integral is taken over the Brillouin zone, and the sum over $a$ goes over all completely occupied bands, assuming there are no partially filled bands.

\footnotetext{
${ }^{4}$ The single-gap approximation is not sufficient for a self-consistent calculation of thermodynamic quantities, such as the free energy and magnetization [41. However, it is adequate for describing low-energy electron states relevant for the QHE.

${ }^{5} \mathrm{~A}$ more rigorous treatment of two periodic potentials is presented in [2 3], using the methods of [42 43 44] and giving the same result (1.16).
} 
Let us apply (1.17) to the FISDW state 1, first setting $Q_{y}=0$. The eigenfunctions of (1.14) are defined on the Brillouin zone torus $-k_{\mathrm{F}} \leq k_{x} \leq$ $k_{\mathrm{F}}$ and $0 \leq k_{y} \leq 2 \pi / b$ with the gap $\Delta \mathrm{e}^{-\mathrm{i} \varphi\left(k_{y}\right)}$ at $k_{x}= \pm k_{\mathrm{F}}$. Let us start with a wave function $\left|\psi_{0}\right\rangle$ at some point $k_{x}^{0}$ far away from $\pm k_{\mathrm{F}}$ and change $k_{x}$ along a closed line encircling the torus at a fixed $k_{y}$. As we pass through $+k_{\mathrm{F}}$, the wave function transforms from $\psi_{+}$to $\mathrm{e}^{\mathrm{i} \varphi} \psi_{-}$. The phase factor appears because the off-diagonal terms in (1.14) have the phase. When we return to the original point $k_{x}^{0}+2 k_{\mathrm{F}}$ in the next Brillouin zone, the wave function becomes $\mathrm{e}^{\mathrm{i} \varphi}\left|\psi_{0}\right\rangle$. The first term in (1.17) is a full derivative in $k_{x}$, so it reduces to a difference taken between $k_{x}^{0}+2 k_{\mathrm{F}}$ and $k_{x}^{0}$

$$
\begin{aligned}
& -\mathrm{i} \frac{e^{2}}{h} \int_{0}^{2 \pi / b} \frac{\mathrm{d} k_{y}}{2 \pi}\left(\left\langle\psi_{0}\left|\mathrm{e}^{-\mathrm{i} \varphi\left(k_{y}\right)} \frac{\partial}{\partial k_{y}} \mathrm{e}^{\mathrm{i} \varphi\left(k_{y}\right)}\right| \psi_{0}\right\rangle-\left\langle\psi_{0}\left|\frac{\partial}{\partial k_{y}}\right| \psi_{0}\right\rangle\right) \\
& =\frac{e^{2}}{h} \int_{0}^{2 \pi / b} \frac{\mathrm{d} k_{y}}{2 \pi} \frac{\partial \varphi\left(k_{y}\right)}{\partial k_{y}}=[\varphi(2 \pi / b)-\varphi(0)] \frac{e^{2}}{h}=\frac{N e^{2}}{h} .
\end{aligned}
$$

Multiplied by the spin factor 2, (1.18) gives the same result as (1.7).

The second term in (1.17) gives zero. In this term, the expression under the integral can be rewritten as the difference $\left.\left\langle\psi\left|\partial_{k_{x}}\right| \psi\right\rangle\right|_{k_{y}=0} ^{k_{y}=2 \pi / b}$. The Hamiltonian (1.14) at $k_{y} b=2 \pi$ is the same as at $k_{y}=0$, and we can select the wave functions to be the same, thus the difference equals zero. It was shown in [1] that the results do not change when we take into account $Q_{y} \neq 0$, $Q_{z} \neq 0$, and the multiple gaps below the Fermi energy generated by the periodic potentials in (1.13).

\subsection{Coexistence of several order parameters}

The formalism presented in Sect.1.5 is particularly useful in the case where several FISDWs with different amplitudes $\Delta_{j}$ and numbers $N_{j}$ coexist [1]. In this case, the off-diagonal terms in (1.14) become

$$
\Delta\left(k_{y}\right)=\sum_{j} \Delta_{j} \exp \left(-i b k_{y} N_{j}\right) .
$$

According to (1.18), the Hall conductivity is determined by the winding number of the complex function (1.19), i.e. by the number of times the phase of $\Delta\left(k_{y}\right)$ changes by $2 \pi$ when $k_{y}$ goes from 0 to $2 \pi / b$. This integer number, taken with the opposite sign, must be substituted in (1.7) instead of $N$. So, when several FISDWs coexist, $\sigma_{x y}$ is not a superposition of partial Hall conductivities, but is always given by the integer winding number of (1.19).

When two FISDWs coexist, $\sigma_{x y}$ is given by the integer $N_{j}$ whose partial gap $\left|\Delta_{j}\right|$ is bigger. To illustrate this, let us use a vector representation of complex numbers and a planetary analogy. Let us associate the first term in 
the sum (1.19) with a vector pointing from the Sun to the Earth, and the second term with a vector from the Earth to the Moon. As the parameter $k_{y}$ increases, the Earth rotates around the Sun, and the Moon rotates around the Earth. The Hall conductivity is determined by the number of times the Moon rotates around the Sun. Clearly, this winding number is determined only by the bigger orbit of the Earth. If one partial gap $\left|\Delta_{l}\right|$ is bigger then the sum of all other partial gaps $\left|\Delta_{l}\right|>\sum_{j \neq l}\left|\Delta_{j}\right|$, then $\sigma_{x y}$ is determined only by the biggest term in (1.19), i.e. $N=N_{l}$ in (1.7).

A FISDW state consisting of multiple periodic potentials was discussed in 47, and the QHE in this model was studied in [4]. Lebed pointed out in 49. that the umklapp scattering requires coexistence of two FISDWs with $N$ and $-N$. The QHE in this case was studied in [50] using the topological method, and it was found that $\sigma_{x y}$ may take the values corresponding to $N,-N$, or zero. A more detailed study was presented in [51]. It was suggested that this effect was may explain sign reversals of the QHE observed in (TMTSF $)_{2} \mathrm{PF}_{6}$ 2733 34. An alternative theory of the QHE sign reversals was proposed in [52. Sign changes of the QHE are also observed in (TMTSF) ${ }_{2} \mathrm{ClO}_{4} \quad 293031$ and (TMTSF $)_{2} \mathrm{ReO}_{4}$ [32, which have anion ordering. Coexistence of the FISDW pairings between different branches of the folded Fermi surface in this case was proposed in [18 17].

\subsection{Temperature evolution of the quantum Hall effect}

Equation (1.16) is a good starting point for discussion of the temperature dependence of the QHE [2] 3, 53. According to this equation, the Hall conductivity can be viewed as the Fröhlich conductivity of the effective 1D density wave (1.14). Thus, the temperature dependence of the QHE must be the same as the temperature dependence of the Fröhlich conductivity, which was studied in the theory of density waves $[54,55$. At $T \neq 0$, the electric current carried by the density-wave condensate is reduced with respect to the zero-temperature value by a factor $f(T)$, which also reduces $\sigma_{x y}$ :

$$
\begin{gathered}
\sigma_{x y}(T)=f(T) 2 N e^{2} / h, \\
f(T)=1-\int_{-\infty}^{\infty} \frac{\mathrm{d} p_{x}}{v_{\mathrm{F}}}\left(\frac{\partial E}{\partial p_{x}}\right)^{2}\left[-\frac{\partial n_{\mathrm{F}}(E / T)}{\partial E}\right],
\end{gathered}
$$

where $E\left(p_{x}\right)$ is the electron dispersion (1.15) in the FISDW state, and $n_{\mathrm{F}}(\epsilon / T)=\left(\mathrm{e}^{\epsilon / T}+1\right)^{-1}$ is the Fermi distribution function with $k_{\mathrm{B}}=1$. Equations (1.20) and (1.21) have a two-fluid interpretation. The first term in (1.21) represents the FISDW condensate current responsible for the QHE.

The second term represents the normal component originating from electron quasiparticles thermally excited above the energy gap. They equilibrate 
with the immobile crystal lattice and do not participate in the Fröhlich current, thus reducing the Hall coefficient. A simple derivation of (1.21) is given in $[2] 3] 53.6$
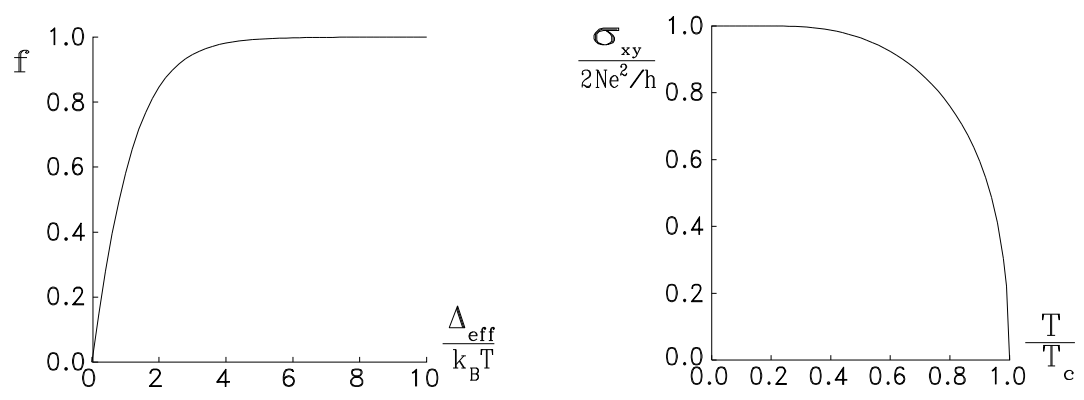

Fig. 1.1. (a) The temperature reduction factor $f(\Delta / T)$ of the Hall conductivity (1.20) given by (1.22). (b) The Hall conductivity (1.20) in the FISDW state as a function of temperature $T$ normalized to the FISDW transition temperature $T_{\mathrm{c}}$

The function $f$ (1.21) depends on $\Delta / T$ and can be written as [8,55]

$$
f\left(\frac{\Delta}{T}\right)=\int_{0}^{\infty} \mathrm{d} \zeta \tanh \left(\frac{\Delta}{2 T} \cosh \zeta\right) / \cosh ^{2} \zeta .
$$

The function $f$ is plotted in Fig.1.1p. It equals 1 at $T=0$, where Eq. (1.20) reproduces the QHE, gradually decreases with increasing $T$, and vanishes at $T \gg \Delta$. Taking into account that the FISDW order parameter $\Delta$ itself depends on $T$ and vanishes at the FISDW transition temperature $T_{\mathrm{c}}$, it is clear that $f(T)$ and $\sigma_{x y}(T)$ vanish at $T \rightarrow T_{\mathrm{c}}$, where $\sigma_{x y}(T) \propto f(T) \propto$ $\Delta(T) \propto \sqrt{T_{\mathrm{c}}-T}$. Assuming that the temperature dependence $\Delta(T)$ is given by the BCS theory [37, 38, we plot $\sigma_{x y}(T)$ in Fig.1.1]. Strictly speaking $\sigma_{x y}(T)$ should not vanish at $T \rightarrow T_{\mathrm{c}}$, but approach to the Hall conductivity of the metallic phase. However, as discussed in Sect.1.2 the Hall effect in the normal state is very small, so this modification is not essential.

The function $f(T)$ (1.21) is qualitatively similar to the function $f_{\mathrm{s}}(T)$ that describes temperature dependence of the superconducting condensate density in the London case [56]. Both functions equal 1 at $T=0$, but the superconducting function behaves differently near $T_{\mathrm{c}}: f_{\mathrm{s}}(T) \propto \Delta^{2}(T) \propto T_{\mathrm{c}}-$ $T$. To understand the difference between the two functions, they should be considered at small, but finite frequency $\omega$ and wave vector $q$ 3. Equations (1.21) and (1.22) represent the dynamic limit, where $q / \omega=0$. This is the relevant limit in our case, because the electric field is homogeneous in space

\footnotetext{
${ }^{6}$ Reference 8 discussed the Hall conductivity in the FISDW state at $T \neq 0$, but failed to reproduce the QHE at $T=0$.
} 
$(q=0)$, but may be time-dependent $(\omega \neq 0)$. The effective periodic potential (1.14) is also time-dependent in the presence of $E_{y}$, as shown in (1.16). On the other hand, for the Meissner effect in superconductors, where the magnetic field is stationary $(\omega=0)$, but varies in space $(q \neq 0)$, the static limit $\omega / q=0$ is relevant. The dynamic and static limits are discussed in more detail in 3 .

In the derivation of (1.20) we assumed that the wave vector $Q_{x}$ (1.4) is always quantized with an integer $N$, so that the energy gap is located at the Fermi level. While this is the case at $T=0$ [57], Lebed pointed out in [58] that $Q_{x}$ is not necessarily quantized at $T \neq 0$. Because of the thermally excited quasiparticles and multiple periodic potentials present in (1.13), the optimal value of $N$ in (1.4) may be non-integer, which results in deviations from the QHE. Equation (1.20) was compared with the experimental temperature dependence of the Hall effect in the FISDW state using a limited data set in [59. and detailed measurements in 60. A good quantitative agreement was found for small integer numbers $N \sim 1$, where the QHE is well-defined. However, for the FISDW with bigger $N$ and lower $T_{\mathrm{c}}$ at lower $B$, poor quantization of the Hall effect was found 61 at the experimentally accessible temperatures, in qualitative agreement with the theory of the non-quantized FISDW [58, 57].

\subsection{Influence of the FISDW motion on the quantum Hall effect}

In the derivation of (1.16), we assumed that the FISDW is pinned and produces only a static periodic potential. However, when the FISDW is subject to a strong or time-dependent electric field, it may move. It is interesting to study how this motion would affect the QHE [2, 3, 62 .

Motion of the FISDW can be described by introducing a time-dependent phase $^{7} \Theta$ of the FISDW amplitude $\Delta_{0}$ in (1.9): $\Delta_{0} \rightarrow \Delta_{0} \mathrm{e}^{-\mathrm{i} \Theta}$. Then, this phase re-appears in the off-diagonal terms in (1.14) and contributes to the electric current (1.16) along the chains

$$
j_{x}=\frac{2 N e^{2}}{h} E_{y}+\frac{2 e}{2 \pi b} \dot{\Theta},
$$

where the dot represents the time derivative. Equation (1.23) needs to be supplemented with an equation of motion for $\Theta$. The latter can be obtained from the effective Lagrangian of the system derived in $62,2,3$

$$
L=-\frac{N e^{2}}{h c} \varepsilon_{i j k} A_{i} F_{j k}+\frac{\hbar}{4 \pi b v_{\mathrm{F}}} \dot{\Theta}^{2}+\frac{e}{\pi b} \Theta E_{x}+\frac{e N}{2 \pi v_{\mathrm{F}}} \dot{\Theta} E_{y} .
$$

Summation of over $(i, j, k)=(x, y, t)$ is implied in the first term, and $F_{j k}$ is the electromagnetic field tensor. The first term in (1.24) is the ChernSimons term responsible for the QHE [63] 1 . The second term is the kinetic

\footnotetext{
${ }^{7}$ The phase $\Theta$ in this paper has the opposite sign to $\Theta$ in [2,3].
} 
energy of a moving FISDW. The third term, well known in the theory of density waves 39 , represents potential energy in the electric field along the chains. The most important for us is the last term, which describes interaction between the FISDW motion and the electric field perpendicular to the chains 62, 2, 3]. This term is permitted by symmetry and has the structure of a mixed product $\boldsymbol{v}[\boldsymbol{E} \times \boldsymbol{B}]$. Here, $\boldsymbol{v}$ is the velocity of the FISDW, proportional to $\dot{\Theta}$ and directed along the chains, i.e. along the $x$ axis. The magnetic field $\boldsymbol{B}$ is directed along the $z$ axis; so, the electric field $\boldsymbol{E}$ enters through the component $E_{y}$. One should keep in mind that the magnetic field enters the last term in (1.24) implicitly, through the integer $N$, which depends on $B$ and changes sign when $B$ changes sign. Varying (1.24) with respect to $\Theta$ and phenomenologically adding the pinning and friction terms, we find the FISDW equation of motion

$$
\ddot{\Theta}+\frac{1}{\tau} \dot{\Theta}+\omega_{0}^{2} \Theta=\frac{2 e v_{\mathrm{F}}}{\hbar} E_{x}-\frac{e N b}{\hbar} \dot{E}_{y},
$$

where $\tau$ is a relaxation time, and $\omega_{0}$ is the pinning frequency.

Let us first consider the ideal case of a free FISDW without pinning and damping. If the electric field $E_{y}$ is applied perpendicular to the chains, the last term in (1.25) induces such a motion of the FISDW that the second term in (1.23) exactly cancels to the first term, and the Hall effect vanishes. If the electric field $E_{x}$ is parallel to the chains, we consider the perpendicular current $j_{y}$ obtained by varying (1.24) with respect to $A_{y}$ :

$$
j_{y}=-\frac{2 N e^{2}}{h} E_{x}+\frac{e N}{2 \pi v_{\mathrm{F}}} \ddot{\Theta} .
$$

Using (1.25) for the ideal case, we see that the two terms in (1.26) cancel out, and the Hall effect vanishes. The cancellation of the QHE by the moving FISDW is in the spirit of Lenz's law, which says that a system responds to an external perturbation in such a way as to minimize its effect. Thus, the QHE exists only if the FISDW is pinned and does not move.

In a more realistic case with pinning and damping, we solve (1.25) by the Fourier transform from time $t$ to frequency $\omega$ and substitute the result into (1.23) and (1.26) to obtain the ac Hall conductivity ${ }^{8}$

$$
\sigma_{x y}(\omega)=\frac{2 N e^{2}}{h} \frac{\omega_{0}^{2}-\mathrm{i} \omega / \tau}{\omega_{0}^{2}-\omega^{2}-\mathrm{i} \omega / \tau} .
$$

The absolute value $\left|\sigma_{x y}\right|$ computed from (1.27) is plotted in Fig.1.2 as a function of $\omega / \omega_{0}$ for $\omega_{0} \tau=2$. It is quantized at $\omega=0$ and has a resonance at the pinning frequency. At higher frequencies, where pinning and damping can be neglected, and the FISDW behaves as a free inertial system, we find that $\sigma_{x y}(\omega) \rightarrow 0$.

\footnotetext{
${ }^{8} \sigma_{x y}(\omega)$ for a FISDW was studied in $[8$, but failed to reproduce the QHE at $\omega=0$.
} 


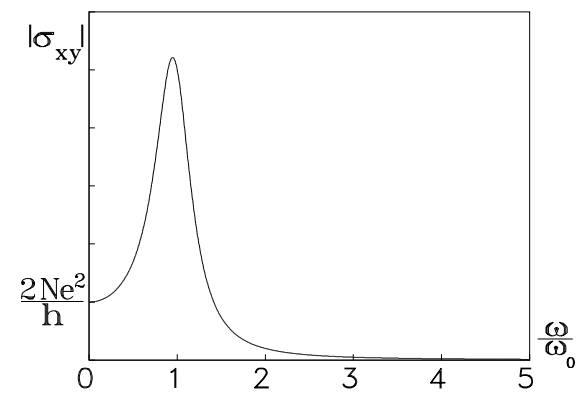

Fig. 1.2. The absolute value of the Hall conductivity $\left|\sigma_{x y}\right|$ computed from (1.27) for $\omega_{0} \tau=2$ as a function of frequency $\omega$ normalized to the pinning frequency $\omega_{0}$

Frequency dependence of the Hall conductivity in semiconducting QHE systems was measured using the technique of crossed wave guides in 64 65. No measurements of $\sigma_{x y}(\omega)$ have been done in (TMTSF) $)_{2} \mathrm{X}$ thus far, but they would be very interesting and can differentiate the QHE in the FISDW state from the conventional QHE in semiconductors. To give a crude estimate of the required frequency range, we quote the pinning frequency $\omega_{0} \sim 3 \mathrm{GHz}$ $\sim 0.1 \mathrm{~K} \sim 10 \mathrm{~cm}$ for a regular SDW (not FISDW) in (TMTSF) ${ }_{2} \mathrm{PF}_{6}$ [6].

The FISDW can be also depinned by a strong dc electric field. In this case, the FISDW motion is controlled by dissipation, which is difficult to study theoretically on microscopic level. For a steady motion, the last terms in (1.25) and (1.26) drop out, so we expect no changes in $\sigma_{x y}$, but some increase in $\sigma_{x x}$ due to the sliding FISDW $80 .{ }^{9}$ If $\sigma_{y y}$ also increases due to increased dissipation and excitation of quasiparticles, then we expect that $\rho_{x x}=\sigma_{y y} /\left(\sigma_{x y}^{2}+\sigma_{x x} \sigma_{y y}\right)$ and $\rho_{y y}=\sigma_{x x} /\left(\sigma_{x y}^{2}+\sigma_{x x} \sigma_{y y}\right)$ would increase when the FISDW starts to slide, whereas $\rho_{x y}=\sigma_{x y} /\left(\sigma_{x y}^{2}+\sigma_{x x} \sigma_{y y}\right)$ would decrease. The experimental measurements in (TMTSF $)_{2} \mathrm{PF}_{6}$ are in qualitative agreement with these expectation [67, although earlier measurements in $(\mathrm{TMTSF})_{2} \mathrm{ClO}_{4}$ 68 produced a different result.

The influence of steady motion of a regular charge-density wave (not FISDW) on the Hall conductivity was studied theoretically in 69 and experimentally in 70,71, 72. In this case, there is no QHE contribution from the condensate, and the effect is primary determined by the thermally excited normal carriers.

\footnotetext{
${ }^{9}$ In principle, due to the presence of $\boldsymbol{B}$, we can phenomenologically add a term proportional to $E_{y}$ to (1.25) and a term proportional to $\dot{\Theta}$ to (1.26). These terms have dissipative origin and violate the time reversal symmetry, so they cannot be obtained from a Lagrangian. Deriving them from the Boltzmann equation is a difficult task.
} 


\subsection{Chiral edge states}

Thus far, we studied the QHE in the bulk. Generally, a system with the integer QHE characterized by the number $N$ is expected to have $N$ chiral edge states 737475 . These electron states are localized at the boundaries of the sample and circulate along the boundary with some velocity $v$, as shown in Fig.1.3 Excitations with the opposite sense of circulation are absent, so these states is chiral. Theory of the edge states in multilayered QHE systems was discussed phenomenologically in 76,77 .

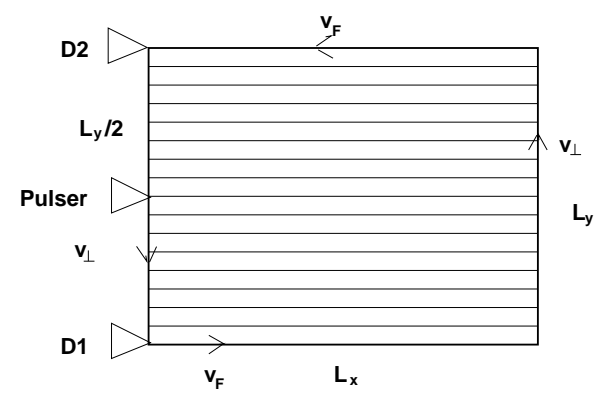

Fig. 1.3. Chiral edge modes circulate around the sample with the velocities $v_{\perp}$ and $v_{\mathrm{F}}$ in the direction of the arrows. The thin parallel lines represent conducting chains of a Q1D material. In the proposed time-of-flight experiment, a pulse from the pulser is detected at different times $t$ and $t^{\prime}$ by the detectors D1 and D2

The QHE can be equivalently formulated in terms of the chiral edge states 73. 74. Suppose a small electric voltage $V_{y}$ is applied across the sample. It produces a difference of chemical potentials between the opposite edges of the sample. The electron states in the bulk of the sample are gapped, so they would not respond to this perturbation. However, the edge modes are gapless, so the difference of chemical potentials produces imbalance $\delta n=$ $2 e V_{y} / h v$ between the occupation numbers of the chiral modes at the opposite edges. Here we utilized the $1 \mathrm{D}$ density of states $2 / h v$, accounting for two spin projections. The chiral modes at the opposite edges propagate in the opposite directions, so the population imbalance between them generates the net edge current $I_{x}$ in the $x$ direction:

$$
I_{x}=e v N \delta n=e v N \frac{2 e V_{y}}{h v}=\frac{2 N e^{2}}{h} V_{y} .
$$

Equation (1.28) represents the QHE, this time for the Hall conductance, rather than conductivity (1.7), which are the same in 2D. Notice that the velocity $v$ of the chiral edge states cancels out in (1.28), and only their number $N$ enters the final formula. The bulk and edge formulations of the QHE are equivalent. Whether the Hall current actually flows in the bulk or along the 
edge depends on where the voltage drops in the sample, but the overall Hall conductance does not depend on this.

Below we discuss the structure of the edge states for the FISDW [2, 4. ${ }^{10}$

\subsubsection{Edges perpendicular to the chains}

First, let us consider a 1D density wave occupying the positive semi-space $x>0$ with an edge at $x=0$. In this case, the wave function (1.8) must vanish at the edge: $\psi(x=0)=0$, so $\psi_{+}(x=0)=-\psi_{-}(x=0)$. With this boundary condition, the Hamiltonian (1.14) also admits a localized electron state, in addition to (1.15), with the energy $|E|<\Delta$ inside the gap:

$$
E=-\Delta \cos \varphi, \quad \psi_{ \pm}= \pm \frac{\mathrm{e}^{-\kappa x}}{\sqrt{\kappa}}, \quad \kappa=-\frac{\sin \varphi}{\xi}, \quad \xi=\frac{\hbar v_{\mathrm{F}}}{\Delta}
$$

The wave function (1.29) exponentially decays into the bulk at a length of the order of the coherence length $\xi$. Equation (1.29) is meaningful only when $\kappa>0$, so the localized state exists on the left edge only for $\pi<\varphi<2 \pi$. However, a solution with $\kappa<0$ is appropriate for the right edge at the opposite end of the sample. The edge state (1.29) is mathematically similar to the localized state at a kink soliton in a density wave 79 .

Now let us consider a FISDW occupying the positive semi-space $x>0$ along the chains and extended in the $y$-direction. In this case, the phase $\varphi=N b k_{y}$ in (1.14) depends on $k_{y}$. Substituting $\varphi\left(k_{y}\right)$ into (1.29), we find 4]

$$
E\left(k_{y}\right)=-\Delta \cos \left(N k_{y} b\right), \quad \kappa=-\frac{\sin \left(N k_{y} b\right)}{\xi}, \quad \psi_{ \pm}= \pm \frac{\mathrm{e}^{\mathrm{i} k_{y} y-\kappa x}}{\sqrt{\kappa}} .
$$

The single bound state (1.29) is now replaced by the band (1.30) of the edge states labeled by the wave vector $k_{y}$ perpendicular to the chains. These states (1.30) are localized along the chains and extended perpendicular to the chains. At the left edge of the sample, we require that $\kappa \propto-\sin \left(N k_{y} b\right)$ is positive, which gives $N$ branches of the edge states in the transverse Brillouin zone $0<k_{y} b<2 \pi$. The complementary $N$ branches of the edge states, determined by the condition $\kappa<0$, exist at the right edge. The dashed and solid lines in Fig.1.4 show the energy dispersion $E\left(k_{y}\right)$ (1.30) of the states localized at the left and right edges for $N=2$. It is clear that the group velocities of the edge states $\partial E\left(k_{y}\right) / \hbar \partial k_{y}$ have opposite signs for the left and right edges. Thus, they carry a surface current around the sample, as indicated by the arrows in Fig. 1.3 The sense of circulation is determined by the sign of $N$, which is controlled by the sign of the magnetic field $B$.

The edge states bands are filled up to the Fermi level in the middle of the gap, and their group velocity at the Fermi level is

\footnotetext{
${ }^{10}$ The edge states in the normal phase of Q1D conductors in a magnetic field were studied in 78 .
} 


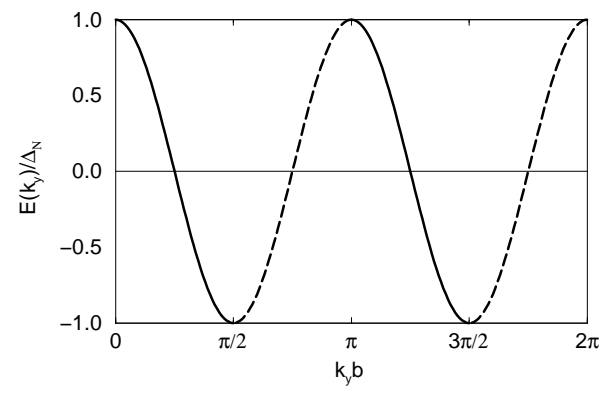

Fig. 1.4. Energy dispersion $E /(-\Delta)$ 1.30 of the electron states localized at the right (solid lines) and left (dashed lines) edges of the sample as a function of the transverse momentum $k_{y}$ for $N=2$

$$
v_{\perp}=\left.\frac{1}{\hbar} \frac{\partial E\left(k_{y}\right)}{\partial k_{y}}\right|_{E=0}=\frac{N b \Delta}{\hbar}
$$

The velocity $v_{\perp}$ is quite low, because it is proportional to the small FISDW gap $\Delta$, so $v_{\perp} \ll v_{\mathrm{F}}$.

\subsubsection{Edges parallel to the chains}

Now let us discuss the edges parallel to the chains. The effective Hamiltonian (1.14) operates on the electron wave functions $\psi_{ \pm}^{\prime}\left(k_{x}, k_{y}\right)$ labeled by the momentum $k_{y}$. The bulk energy spectrum (1.15) of (1.14) is degenerate in $k_{y}$. Thus, we can use the Wannier wave functions $\psi_{ \pm}^{\prime}\left(k_{x}, M\right)=$ $\int \mathrm{e}^{\mathrm{i} k_{y} M b} \psi_{ \pm}^{\prime}\left(k_{x}, k_{y}\right) \mathrm{d} k_{y} / 2 \pi$ as a new basis 8078 . The wave function $\psi_{ \pm}^{\prime}\left(k_{x}, M\right)$ is localized across the chains around the chain with the number $M$. Introducing the destruction operators $\hat{a}_{ \pm}\left(p_{x}, M\right)$ for this basis, we can rewrite the Hamiltonian (1.14) in the following form 80.

$$
\begin{aligned}
& \hat{H}^{\prime}=\int \frac{\mathrm{d} k_{x}}{2 \pi} \sum_{M} v_{\mathrm{F}} p_{x}\left[\hat{a}_{+}^{+}\left(p_{x}, M\right) \hat{a}_{+}\left(p_{x}, M\right)-\hat{a}_{-}^{+}\left(p_{x}, M\right) \hat{a}_{-}\left(p_{x}, M\right)\right] \\
& +\Delta\left[\hat{a}_{+}^{+}\left(p_{x}, M+N\right) \hat{a}_{-}\left(p_{x}, M\right)+\hat{a}_{-}^{+}\left(p_{x}, M\right) \hat{a}_{+}\left(p_{x}, M+N\right)\right] .
\end{aligned}
$$

As a consequence of the $k_{y}$-dependent phase in the off-diagonal terms in (1.14), the Hamiltonian (1.32) represents pairing between the $+k_{\mathrm{F}}$ and $-k_{\mathrm{F}}$ states localized at the different chains $M+N$ and $M$. For the chains in the bulk of the crystal, this pairing results in the gapped energy spectrum (1.15). However, the states at the edges are exceptional. The $+k_{\mathrm{F}}$ states on the first $N$ chains on one side of the crystal and the $-k_{\mathrm{F}}$ states on the last $N$ chains on the other side of the crystal do not have partners to couple with, so these states remain ungapped [2]. Thus, one side of the sample possesses $N$ gapless chiral modes propagating along the edge with the velocity $+v_{\mathrm{F}}$, and the other 
side has $N$ gapless chiral modes propagating in the opposite direction with the velocity $-v_{F}$, as shown in Fig.1.3.

\subsubsection{Possibilities for experimental observation of the chiral edges states}

Let us estimate parameters of the chiral edge states. The activation energy in the FISDW state with $N=1$ was found to be $2 \Delta=6 \mathrm{~K}$ in (TMTSF) ${ }_{2} \mathrm{ClO}_{4}$ at $B=25 \mathrm{~T}$ [81. Substituting $\Delta=3 \mathrm{~K}$ and $N=1$ into (1.31), we find $v_{\perp}=300 \mathrm{~m} / \mathrm{s}$, which is three orders of magnitude lower than $v_{\mathrm{F}}=10^{5} \mathrm{~m} / \mathrm{s}$ [6]. Despite the big difference in velocities, the total currents of the parallel and perpendicular edge states are the same. Indeed, the slow perpendicular states with $v_{\perp}=N b \Delta / \hbar$ have the large width $\xi=\hbar v_{\mathrm{F}} / \Delta$, whereas the fast parallel states with the velocity $v_{\mathrm{F}}$ have the narrow width $N b$. The total edge current $I$ carried by the perpendicular states (1.30) is

$$
I=\frac{2 N e}{h} \int_{0}^{\pi / 2 b N} \frac{\partial E\left(k_{y}\right)}{\partial k_{y}} \mathrm{~d} k_{y}=\frac{2 N e \Delta}{h}=\frac{e v_{\perp}}{\pi b}=20 \mathrm{nA} .
$$

The same current is carried along the chains by the difference between the gapped and ungapped branches of the electron dispersion (1.15)

$$
I=\frac{2 N e}{h} \int_{-\infty}^{0}\left(\frac{\partial E\left(p_{x}\right)}{\partial p_{x}}-v_{\mathrm{F}}\right) \mathrm{d} p_{x}=\frac{2 N e \Delta}{h} .
$$

It is tempting to use (1.33) to calculate magnetization of the sample. However, there are additional contributions to the total magnetization coming from the edge states inside the energy gaps opened by the neglected terms in (1.13) below the Fermi level. Magnetization of the FISDW state was calculated in [41] using the bulk free energy and was measured in (TMTSF $)_{2} \mathrm{ClO}_{4}$ in [15].

The most convincing demonstration of the edge states would be the timeof-flight experiment [4] analogous to that performed in GaAs in [82 83]. The experimental setup is sketched in Fig.1.3 An electric pulse is applied by the pulser at the center of the edge perpendicular to the chains. The pulse travels counterclockwise around the sample. For the typical sample dimensions $L_{x}=$ $2 \mathrm{~mm}$ and $L_{y}=0.2 \mathrm{~mm}$, we find the times of flight $t_{x}=L_{x} / v_{\mathrm{F}}=8 \mathrm{~ns}$ and $t_{y}=L_{y} / v_{\perp}=0.67 \mu$ s using the values of $v_{\perp}$ and $v_{\mathrm{F}}$ quoted above for $N=1$. Thus, the pulse will reach the detector D1 at the time $t=L_{y} / 2 v_{\perp}$ and the other detector $\mathrm{D} 2$ at the longer time $t^{\prime}=3 t+2 L_{x} / v_{\mathrm{F}} \approx 3 t$. The difference between the arrival times $t$ and $t^{\prime}$ is a signature of the chiral edge states. The flight time $t$ should exhibit discontinuities at the FISDW phase boundaries due to discontinuity of both $N$ and $\Delta$ affecting $v_{\perp}$ in (1.31). The pulse must be shorter than $t=0.33 \mu$ s for clear resolution and longer than $\hbar / \Delta=2.6$ ps, so that only the low-energy excitations are probed. 
Existence of the edge states was confirmed in a multilayered GaAs system by showing that the conductance $G_{z z}$ perpendicular to the layers is proportional to the number of the edge states and the perimeter of the sample 84]. A similar experiment in (TMTSF $)_{2} \mathrm{AsF}_{6}$ produced inconclusive results 85.

The specific heat per layer $C_{\mathrm{e}}$ of the gapless edge excitations is proportional to temperature at $T \ll \Delta[4$ :

$$
\frac{C_{\mathrm{e}}}{T}=\frac{N \pi}{3 \hbar}\left(\frac{2 L_{y}}{v_{\perp}}+\frac{2 L_{x}}{v_{\mathrm{F}}}\right) \approx \frac{2 \pi}{3 \Delta} \frac{L_{y}}{b} \approx 3 \times 10^{-3} \frac{\mathrm{mJ}}{\mathrm{K}^{2} \text { mole }},
$$

where the dominant contribution comes from the edges perpendicular to the chains. The ratio of $C_{\mathrm{e}} / T$ (1.35) to the bulk specific heat in the normal state $C_{\mathrm{b}}^{(\mathrm{n})} / T=\pi L_{x} L_{y} / 3 \hbar b v_{\mathrm{F}}$ is roughly equal to the ratio of the volumes occupied by the bulk and edge states: $C_{\mathrm{b}}^{(\mathrm{n})} / C_{\mathrm{e}}=L_{x} / 2 \xi \approx 10^{3}$. The experimentally measured $C_{\mathrm{b}}^{(\mathrm{n})} / T$ is $5 \mathrm{~mJ} /\left(\mathrm{K}^{2}\right.$ mole $)$ [17. In the FISDW state, the bulk specific heat is exponentially suppressed because of the energy gap $\Delta$ and becomes smaller than $C_{\mathrm{e}}$ (1.35) at sufficiently low temperatures $T \leq \Delta / 14$ [4]. This regime could have been possibly achieved in the specific heat measurements [17 performed at $T=0.32 \mathrm{~K}$ and $B=9 \mathrm{~T}$ in (TMTSF) ${ }_{2} \mathrm{ClO}_{4}$. According to (1.35), $C_{\mathrm{e}} / T$ must be discontinuous at the boundaries between the FISDW phases, where $\Delta$ changes discontinuously 41]. Notice that $N$ cancels out in (1.35), in contrast to the phenomenological model [77.

Other possibilities for experimental observation of the edge states are discussed in [86]. We would like to mention that non-chiral midgap edge states are expected to exist in the superconducting $p$-wave state of (TMTSF $)_{2} \mathrm{X}$ [87.

\subsection{Generalization to the three-dimensional quantum Hall effect}

Experiments show that the FISDW state in (TMTSF) ${ }_{2} \mathrm{X}$ depends only on the $B_{z}$ component of a magnetic field. Thus, although the (TMTSF) $)_{2} \mathrm{X}$ crystals are three-dimensional (3D), they can be treated as a collection of essentially independent 2D layers, and the QHE can be studied within a 2D theory. Nevertheless, let us discuss a generalization of this theory to the 3D case.

Suppose a magnetic field has the $B_{z}$ and $B_{y}$ components perpendicular to the chains. As Lebed pointed out in 88, inserting them into (1.1) via the Peierls-Onsager substitution creates two periodic potentials in the $x$ direction, $2 t_{b} \cos \left(k_{y} b-G_{1} x\right)$ and $2 t_{c} \cos \left(k_{z} d+G_{2} x\right)$, with the wave vectors $G_{1}$ and $G_{2}$. Thus, a FISDW may form with the wave vector 89]

$$
Q_{x}=2 k_{\mathrm{F}}-N_{1} G_{1}-N_{2} G_{2}, \quad G_{1}=e b B_{z} / \hbar c, \quad G_{2}=e d B_{y} / \hbar c .
$$

Repeating the derivation of Sect.1.4 and introducing the electric field components $E_{x}$ and $E_{y}$ as in (1.16), we find the electric current per one chain 90 91] 


$$
I_{x}=\frac{2 e}{2 \pi} \frac{\partial \varphi}{\partial t}=\frac{2 e^{2}}{h}\left(N_{1} b E_{y}-N_{2} d E_{z}\right) .
$$

Using the current density per unit area $j_{x}=I_{x} / b d$, we rewrite (1.37) as

$$
\boldsymbol{j}=\frac{2 e^{2}}{h} \boldsymbol{E} \times \boldsymbol{K}, \quad \boldsymbol{K}=\left(0, \frac{N_{2}}{b}, \frac{N_{1}}{d}\right) .
$$

Equation (1.38) with an integer vector $\boldsymbol{K}$ belonging to the reciprocal crystal lattice represents the general form of the QHE in a 3D system 92. This formula was applied to the 3D FISDW (1.36) in [89, 91, 93, and to general lattices in 94, 95, 96. The edge states picture, presented in Sect.1.9 was generalized to the 3D FISDW in 97.

Although the 3D FISDW (1.36) does not realize in (TMTSF) $)_{2} \mathrm{X}$, it may occur in other families of Q1D materials. It would be very interesting to investigate whether FISDW exists in the Q1D material (DI-DCNQI) 2 Ag, where $t_{b}=t_{c}$ by crystal symmetry, so there is no layered structure [98.

\subsection{Conclusions and open questions}

In summary, the QHE in the FISDW state is a direct consequence of the quantization and magnetic field dependence of the wave vector $Q_{x}$ (1.4). As a result, $N$ completely filled Landau bands are maintained between $Q_{x}$ and $2 k_{\mathrm{F}}$, whereas the Fermi sea plays the role of a reservoir. More rigorously, the QHE can be obtained as a topological invariant in terms of the winding number of a phase in the Brillouin zone. The topological approach is particularly useful when several FISDWs coexist. The Hall effect can be also viewed as the Fröhlich current of an effective density wave. ${ }^{11}$ This allows us to derive its temperature dependence within a two-fluid picture. Motion of the FISDW cancels the Hall effect in the ideal case of a free FISDW and at high frequency. The QHE can be also formulated in terms of the chiral edge states circulating around the sample with low speed across the chains and high speed along the chains. The QHE can be also generalized for a three-dimensional FISDW. While the temperature dependence of the QHE was measured experimentally and found to be in agreement with the theory, the other theoretical results, such as the frequency dependence of $\sigma_{x y}$ and the existence of the edge states, await experimental verification. It would be interesting to search for a $3 \mathrm{D}$ FISDW in (DI-DCNQI) $)_{2} \mathrm{Ag}$.

Below we list some open questions in the theory of the QHE and the FISDW. One problems is that, in experiment, the dissipative components $\sigma_{x x}$ and $\sigma_{y y}$ tend to saturate at small, but finite values in the limit $T \rightarrow 0$ 2728]. The reason for this is not completely clear, but may be due to impurity

\footnotetext{
${ }^{11}$ The theory presented in Sect.1.4 and [1.16) was also applied to quantized adiabatic transport induced by surface acoustic waves in carbon nanotubes [99.
} 
scattering [100]. As a result, the Hall effect quantization is not as good as in semiconducting systems, especially at lower magnetic fields.

Predicting the sequence of $N$ as a function of $B$ is a task for the FISDW theory. For a simple model, the theory gives consecutive numbers [21,41], as observed experimentally at higher pressures in (TMTSF) ${ }_{2} \mathrm{PF}_{6}$ [25,26, 27, 28]. However, at lower pressures, a complicated sequence of $N$ with multiple sign reversals and a bifurcation in the $B-T$ phase diagram is observed 33 34. This sequence is not fully understood, although theoretical attempts have been made [49, $48,51,52,50$.

Developing a detailed theory of the FISDW in (TMTSF) ${ }_{2} \mathrm{ClO}_{4}$ and (TMTSF $)_{2} \mathrm{ReO}_{4}$ is even more difficult because of the period doubling in the $\boldsymbol{b}$ direction due to the anion ordering in these materials. Experiments show a complicated phase diagram for (TMTSF $)_{2} \mathrm{ClO}_{4}$ [18, 17. Numerous theoretical scenarios for the FISDW in (TMTSF) ${ }_{2} \mathrm{ClO}_{4}$ are reviewed by S. Haddad et al. in this book and in 101. Particularly puzzling is the behavior of (TMTSF $)_{2} \mathrm{ClO}_{4}$ in very strong magnetic fields between 26 and $45 \mathrm{~T}$, where it is supposed to be in the FISDW phase with $N=0$, i.e. with zero Hall effect. Instead, the Hall coefficient and other quantities show giant oscillations as a function of the magnetic field [102. This phenomenon is not fully understood, but may be related to the soliton theory [103].

\section{References}

1. V.M. Yakovenko: Phys. Rev. B 43, 11353 (1991)

2. V.M. Yakovenko, H.-S. Goan: J. Phys. I (France) 6, 1917 (1996)

3. V.M. Yakovenko, H.S. Goan: Phys. Rev. B 58, 10648 (1998)

4. K. Sengupta, H.-J. Kwon, V.M. Yakovenko: Phys. Rev. Lett. 86, 1094 (2001)

5. T. Ishiguro, K. Yamaji, G. Saito: Organic Superconductors, 2nd edn (Springer, Berlin, 1998)

6. S. Takahashi, S. Hill, S. Takasaki, J. Yamada, H. Anzai: Phys. Rev. B 72, $024540(2005)$

7. J.R. Cooper, M. Miljak, G. Delplanque, D. Jérome, M. Weger, J.M. Fabre, L. Giral: J. Phys. (France) 38, 1097 (1977); J.R. Cooper, B. Korin-Hamzić in Organic Conductors, ed by J.-P. Farges (Dekker, New York, 1994) p 359

8. A. Virosztek, K. Maki: Phys. Rev. B 39, 616 (1989)

9. V.M. Yakovenko, A.T. Zheleznyak: Synth. Metals 103, 2202 (1999)

10. G. Mihály, I. Kézsmárki, F. Zámborszky, L. Forró: Phys. Rev. Lett. 84, 2670 (2000)

11. J. Moser, J.R. Cooper, D. Jérome, B. Alavi, S.E. Brown, K. Bechgaard: Phys. Rev. Lett. 84, 2674 (2000)

12. V.M. Yakovenko, A.T. Zheleznyak: Synth. Metals 120, 1083 (2001)

13. F. Pesty, P. Garoche, K. Bechgaard: Phys. Rev. Lett. 55, 2495 (1985)

14. N.A. Fortune, J.S. Brooks, M.J. Graf, G. Montambaux, L.Y. Chiang, J.A.A.J. Perenboom, D. Althof: Phys. Rev. Lett. 64, 2054 (1990)

15. M.J. Naughton, J.S. Brooks, L.Y. Chiang, R.V. Chamberlin, P.M. Chaikin: Phys. Rev. Lett. 55, 969 (1985) 
16. T. Takahashi, D. Jérome, K. Bechgaard: J. Phys. (France) 45, 945 (1984)

17. U.M. Scheven, E.I. Chashechkina, E. Lee, P. M. Chaikin: Phys. Rev. B 52, 3484 (1995)

18. S.K. McKernan, S.T. Hannahs, U.M. Scheven, G.M. Danner, P.M. Chaikin: Phys. Rev. Lett. 75, 1630 (1995)

19. I.F. Schegolev Memorial Issue: J. Phys. I (France) 6, December issue \#12 (1996)

20. M. Héritier, G. Montambaux, P. Lederer: J. Phys. Lett. (France) 45, L943 (1984)

21. G. Montambaux, M. Héritier, P. Lederer: Phys. Rev. Lett. 55, 2078 (1985); Erratum 56, 97 (1986)

22. L.P. Gor'kov: Sov. Phys. Usp. 27, 809 (1985)

23. A.G. Lebed: Sov. Phys. JETP 62, 595 (1985)

24. D. Poilblanc, G. Montambaux, M. Héritier, P. Lederer: Phys. Rev. Lett. 58, 270 (1987)

25. P.M. Chaikin, W. Kang, S. Hannahs, R.C. Yu: Physica B 177, 353 (1992)

26. S.T. Hannahs, J.S. Brooks, W. Kang, L.Y. Chiang, P.M. Chaikin: Phys. Rev. Lett. 63, 1988 (1988)

27. J.R. Cooper, W. Kang, P. Auban. G. Montambaux, D. Jérome, K. Bechgaard: Phys. Rev. Lett. 63, 1984 (1988)

28. S. Valfells, J.S. Brooks, Z. Wang, S. Takasaki, J. Yamada, H. Anzai, M. Tokumoto: Phys. Rev. B 54, 16413 (1996)

29. R.V. Chamberlin, M.J. Naughton, X. Yan, L.Y. Chiang, S.-Y. Hsu, P.M. Chaikin: Phys. Rev. Lett. 60, 1189 (1988)

30. M.J. Naughton, R.V. Chamberlin, X. Yan, S.-Y. Hsu, L.Y. Chiang, M.Ya. Azbel, P.M. Chaikin: Phys. Rev. Lett. 61, 621 (1988)

31. W. Kang, D. Jérome: J. Phys. I (France) 1, 449 (1991)

32. W. Kang, J.R. Cooper, D. Jérome: Phys. Rev. B 43, 11467 (1991)

33. L. Balicas, G. Kriza, F.I.B. Williams, Phys. Rev. Lett. 75, 2000 (1995)

34. H. Cho, W. Kang: Phys. Rev. B 59, 9814 (1999)

35. L.P. Gor'kov, A.G. Lebed: J. Phys. Lett. (France) 45, L433 (1984)

36. P.M. Chaikin: Phys. Rev. B 31, 4770 (1985)

37. D. Poilblanc, M. Héritier, G. Montambaux, P. Lederer: J. Phys. C 19, L321 (1986)

38. A. Virosztek, L. Chen, K. Maki: Phys. Rev. B 34, 3371 (1986)

39. G. Grüner: Density Waves in Solids (Addison-Wesley, New York, 1994)

40. S.-R. Chang, K. Maki: J. Low Temp. Phys. 66, 357 (1987)

41. G. Montambaux, D. Poilblanc: Phys. Rev. B 37, 1913 (1988); G. Montambaux, M. J. Naughton, R. V. Chamberlin, X. Yan, P. M. Chaikin, M. Ya. Azbel: Phys. Rev. B 39, 885 (1989)

42. I. Dana, Y. Avron, J. Zak: J. Phys. C 18, L679 (1985)

43. H. Kunz: Phys. Rev. Lett. 57, 1095 (1986)

44. M. Kohmoto: J. Phys. Soc. Jpn. 62, 659 (1993)

45. D.J. Thouless in The Quantum Hall Effect, ed by R.E. Prange, S.M. Girvin (Springer, Berlin, 1987) Chap 4

46. M. Kohmoto: Phys. Rev. B 39, 11943 (1989)

47. K. Machida, M. Nakano: J. Phys. Soc. Jpn. 59, 4223 (1990); K. Machida, Y. Hori, M. Nakano: J. Phys. Soc. Jpn. 60, 1730 (1991); Y. Hori, K. Machida: J. Phys. Soc. Jpn. 61, 1246 (1992); K. Machida, Y. Hori, M. Nakano: Phys. Rev. Lett. 70, 61 (1993) 
48. K. Machida, Y. Hasegawa, M. Kohmoto, V.M. Yakovenko, Y. Hori, K. Kishigi: Phys. Rev. B 50, 921 (1994); Y. Hasegawa, K. Machida, M. Kohmoto, V.M. Yakovenko: J. Supercond. 7, 757 (1994)

49. A. G. Lebed: JETP Lett. 51, 663 (1990); Physica B 169, 368 (1991); Sov. Phys. JETP 72, 1035 (1991), Phys. Scripta T 39, 386 (1991)

50. N. Dupuis, V. M. Yakovenko: Phys. Rev. Lett. 80, 3618 (1998)

51. N. Dupuis, V. M. Yakovenko: Phys. Rev. B 58, 8773 (1998); Europhys. Lett. 45, 361 (1999); Phys. Rev. B 61, 12888 (2000)

52. D. Zanchi, G. Montambaux, Phys. Rev. Lett. 77, 366 (1996)

53. H.-S. Goan, V. M. Yakovenko: Synth. Metals 85, 1609 (1997)

54. P.A. Lee, T.M. Rice: Phys. Rev. B 19, 3970 (1979); T.M. Rice, P.A. Lee, M.C. Cross: Phys. Rev. B 20, 1345 (1979)

55. K. Maki, A. Virosztek: Phys. Rev. B 41, 557 (1990); 42, 655 (1990)

56. J. R. Schrieffer: Theory of Superconductivity, 2nd edn (Perseus Books, Reading, 1999) p 218

57. K. Sengupta, N. Dupuis: Phys. Rev. B 68, 094431 (2003)

58. A.G. Lebed: Phys. Rev. Lett. 88, 177001 (2002); JETP Lett. 72, 141 (2000)

59. V.M. Yakovenko, H.-S. Goan, J. Eom, W. Kang: J. Phys. IV (France) 9, Pr10-195 (1999)

60. T. Vuletić, C. Pasquier, P. Auban-Senzier, S. Tomić, D. Jérome, K. Maki, K. Bechgaard: Eur. Phys. J. B 21, 53 (2001)

61. A.V. Kornilov, V.M. Pudalov, Y. Kitaoka, K. Ishida, T. Mito, J.S. Brooks, J.S. Qualls, J.A.A.J. Perenboom, N. Tateiwa, T.C. Kobayashi: Phys. Rev. B 65, 060404 (2002)

62. V.M. Yakovenko: J. Phys. IV (France), Colloque C2, 3, 307 (1993); J. Supercond. 7, 683 (1994)

63. V.M. Yakovenko: Phys. Rev. Lett. 65, 251 (1990)

64. F. Kuchar, R. Meisels, G. Weimann, W. Schlapp: Phys. Rev. B 33, 2965 (1986)

65. L.A. Galchenkov, I.M. Grodnenskii, M.V. Kostovetskii, O. R. Matov: JETP Lett. 46, 542 (1987)

66. D. Quinlivan, Y. Kim, K. Holczer, G. Grüner, F. Wudl: Phys. Rev. Lett. 65, 1816 (1990)

67. L. Balicas: Phys. Rev. Lett. 80, 1960 (1998)

68. T. Osada, N. Miura, I. Oguro, G. Saito: Phys. Rev. Lett. 58, 1563 (1987)

69. S.N. Artemenko, A.N. Kruglov: Sov. Phys. Solid State 26, 1448 (1984); E.N. Dolgov, Sov. J. Low Temp. Phys. 10, 747 (1984)

70. G.X. Tessema, N.P. Ong: Phys. Rev. B 23, 5607 (1981)

71. S.N. Artemenko, E.N. Dolgov, A.N. Kruglov, Yu.I. Latshyev, Ya.S. Savitskaya, V.V. Frolov: JETP Lett. 46, 891 (1984)

72. L. Forró, J.R. Cooper, A. Jánossy, K. Kamarás: Phys. Rev. B 34, 9047 (1986); L. Forró, J.R. Cooper, A. Jánossy, M. Maki: Solid State Commun. 62, 715 (1987); M. Petravić, L. Forró, J.R. Cooper, F. Levy: Phys. Rev. B 40, 2885 (1989)

73. B.I. Halperin: Phys. Rev. B 25, 2185 (1982)

74. C.L. Kane, M.P.A. Fisher in Perspectives in Quantum Hall Effects, ed by S. Das Sarma, A. Pinczuk (Wiley, New York, 1997) Chap 4.

75. Y. Hatsugai: Phys. Rev. Lett. 71, 3697 (1993); Phys. Rev. B 48, 11851 (1993); J. Phys. Cond. Mat. 9, 2507 (1997) 
76. J.T. Chalker, A. Dohmen: Phys. Rev. Lett. 75, 4496 (1995)

77. L. Balents, M.P.A. Fisher: Phys. Rev. Lett. 76, 2782 (1996)

78. V.M. Yakovenko, H.-S. Goan: Phys. Rev. B 58, 8002 (1998)

79. S.A. Brazovskii: Sov. Phys. JETP 51, 342 (1980)

80. V.M. Yakovenko: Europhys. Lett. 3, 1041 (1987); Sov. Phys. JETP 66, 355 (1987)

81. R.V. Chamberlin, M.J. Naughton, X. Yan, L.Y. Chiang, S.-Y. Hsu, P.M. Chaikin: Synth. Metals 27, 41 (1988)

82. R.C. Ashoori, H.L. Stormer, L.N. Pfeiffer, K.W. Baldwin, K. West: Phys. Rev. B 45, 3894 (1992)

83. G. Ernst, R.J. Haug, J. Kuhl, K. von Klitzing, K. Eberl: Phys. Rev. Lett. 79, 3748 (1997)

84. D.P. Druist, P.J. Turley, K.D. Maranowski, E.G. Gwinn, A.C. Gossard: Phys. Rev. Lett. 80, 365 (1998)

85. S. Uji, C. Terakura, M. Takashita, T. Terashima, H. Aoki, J.S. Brooks, S. Tanaka, S. Maki, J. Yamada, S. Nakatsuji: Phys. Rev. B 60, 1650 (1999)

86. H.-J. Kwon, V.M. Yakovenko, K. Sengupta: Synth. Metals 133-134, 27 (2003)

87. K. Sengupta, I. Zutić, H.-J. Kwon, V.M. Yakovenko, S. Das Sarma: Phys. Rev. B 63, 144531 (2001)

88. A.G. Lebed: JETP Lett. 43, 174 (1986)

89. G. Montambaux, P.B. Littlewood: Phys. Rev. Lett. 62, 953 (1989); Erratum 63, 818 (1989)

90. Y. Sun, K. Maki: Phys. Rev. B 49, 15356 (1994)

91. Y. Hasegawa: Phys. Rev. B 51, 4306 (1995)

92. B.I. Halperin: Jap. J. Appl. Phys. Suppl. 26-3, 1913 (1987)

93. M. Koshino, H. Aoki, T. Osada: Phys. Rev. B 65, 205311 (2002)

94. G. Montambaux, M. Kohmoto: Phys. Rev. B 41, 11417 (1990)

95. M. Kohmoto, B.I. Halperin, Y.-S. Wu: Phys. Rev. B 45, 13488 (1992)

96. M. Koshino, H. Aoki, K. Kuroki, S. Kagoshima, T. Osada: Phys. Rev. Lett. 86, 1062 (2001); Phys. Rev. B 65, 045310 (2002)

97. M. Koshino, H. Aoki, B.I. Halperin: Phys. Rev. B 66, 081301 (2002)

98. T. Itou, K. Kanoda, K. Murata, T. Matsumoto, K. Hiraki, T. Takahashi: Phys. Rev. Lett. 93, 216408 (2004); Phys. Rev. B 72, 113109 (2005)

99. V.I. Talyanskii, D.S. Novikov, B.D. Simons, L.S. Levitov: Phys. Rev. Lett. 87, 276802 (2001); P.J. Leek, M.R. Buitelaar, V.I. Talyanskii, C.G. Smith, D. Anderson, G.A.C. Jones, J. Wei, D.H. Cobden: Phys. Rev. Lett. 95, 256802 (2005)

100. M.Ya. Azbel, P. Bak, P.M. Chaikin: Phys. Rev. Lett. 59, 926 (1987)

101. S. Haddad, S. Charfi-Kaddour, M. Héritier, R. Bennaceur: Phys. Rev. B 72, $085104(2005)$

102. S. Uji, S. Yasuzuka, T. Konoike, K. Enomoto, J. Yamada, E.S. Choi, D. Graf, J.S. Brooks: Phys. Rev. Lett. 94, 077206 (2005)

103. A.G. Lebed: Phys. Rev. B 55, 1299 (1997) 